Association for Information Systems AIS Electronic Library (AISeL)

AMCIS 2004 Proceedings

Americas Conference on Information Systems

(AMCIS)

December 2004

\title{
A Framework For Assessing E-Health Preparedness
}

Nilmini Wickramasinghe

Cleveland State University

Adam Fadlalla

Cleveland State University

Elie Geisler

Illinois Institute of Technology

Jonathan Schaffer

The Cleveland Clinic

Follow this and additional works at: http://aisel.aisnet.org/amcis2004

\section{Recommended Citation}

Wickramasinghe, Nilmini; Fadlalla, Adam; Geisler, Elie; and Schaffer, Jonathan, "A Framework For Assessing E-Health Preparedness" (2004). AMCIS 2004 Proceedings. 26.

http://aisel.aisnet.org/amcis2004/26

This material is brought to you by the Americas Conference on Information Systems (AMCIS) at AIS Electronic Library (AISeL). It has been accepted for inclusion in AMCIS 2004 Proceedings by an authorized administrator of AIS Electronic Library (AISeL). For more information, please contact elibrary@aisnet.org. 


\title{
A FRAMEWORK FOR ASSESSING E-HEALTH PREPAREDNESS
}

\author{
Nilmini S. Wickramasinghe \\ Cleveland State University \\ Cleveland, OH 44114, USA \\ n.wickramasinghe@csuohio.edu
}

\author{
Adam M.A. Fadlalla \\ Cleveland State University \\ Cleveland, OH 44114, USA \\ a.fadlalla@csuohio.edu
}

\author{
Elie Geisler \\ Illinois Institute of Technology \\ 565 W Adams Street, M/C 294 \\ Chicago, Illinois 60607, USA \\ geisler@mail.stuart.iit.edu \\ Jonathan L. Schaffer \\ The Cleveland Clinic \\ 9500 Euclid Avenue, Desk A41 \\ Cleveland, OH 44195, USA \\ schaffj@ccf.org
}

\begin{abstract}
While healthcare is the biggest service industry on the globe it has yet to realize the full potential of the e-business revolution in the form of e-health. This is due to many reasons including the fact that the healthcare industry is faced with many complex challenges in trying to deliver cost-effective, high-value, accessible healthcare and has traditionally been slow to embrace new business techniques and technologies. Given that e-health to a great extent is a macro level concern that has far reaching micro level implications, this paper firstly develops a framework to assess a country's preparedness with respect to embracing e-health (the application of e-commerce to healthcare) and from this an e-health preparedness grid to facilitate the assessment of any e-health initiative. Taken together the integrative framework and preparedness grid provide useful and necessary tools to enable successful e-health initiatives to ensue by helping country and/or organization within a country to identify and thus address areas that require further attention in order for it to undertake a successful e-health initiative.
\end{abstract}

KEYWORDS: healthcare, e-health, telemedicine, evidence-based medicine, infrastructure, framework.

\section{INTRODUCTION}

Technology has the potential to help solve many of the problems faced by developed and developing countries alike; from improving healthcare delivery to opening up commerce opportunities. The numbers of nations offering e-commerce solutions are increasing every year, and consequently the number of workplaces that have Internet connection for business activities has been rapidly growing worldwide. Table 1 provides three estimates of e-commerce forecasts. These projections clearly highlight the important role of e-commerce on the global economy. However, as the Secretary General of The United Nations notes in his forward to the e-commerce and development report [UNCTAD, 2002] " knowing that an instrument is powerful is not enough to ensure that it will be put to the best use. We need to understand how it works, and how and when it should be used ... and maximize its power."

Within the umbrella of e-commerce one area, e-health has yet to reach its full potential in many developed countries, let alone developing countries. Each country is positioned differently and has varying potential and preparedness regarding embracing e-commerce technologies generally and e-health in particular. Given the macro level nature of many issues pertaining to the development of e-health [Alvarez, 2002], in order to be more effective in their e-health initiatives, it is important for countries to assess their potential, identify their relative strengths and weaknesses and thereby develop strategies and policies to address these issues to effectively formulate and implement appropriate e-health initiatives. To do this effectively, it is valuable to have an integrative framework that enables the assessment of a country's e-health preparedness. This paper serves to develop such a framework that can be applied to various countries throughout the globe 
and from this to generate an e-health preparedness grid. In so doing, we hope to facilitate better understanding of e-health initiatives and thus maximize their power.

\begin{tabular}{|l|lllllll|} 
Source & 2000 & 2001 & 2002 & 2003 & 2004 & 2005 & 2006 \\
Forester & & & $\mathbf{2 , 2 9 3 . 5 0}$ & $\mathbf{3 , 8 7 8 . 8 0}$ & $\mathbf{6 , 2 0 1 . 1 0}$ & $\mathbf{9 , 2 4 0 . 6 0}$ & $\mathbf{1 2 , 8 3 7 . 3 0}$ \\
\hline IDC & $\mathbf{3 5 4 . 9 0}$ & $\mathbf{6 1 5 . 3 0}$ & & & & $\mathbf{4 , 6 0 0 . 0 0}$ & \\
& & & & & & & \\
Emarketer & $\mathbf{2 7 8 . 1 9}$ & $\mathbf{4 7 4 . 3 2}$ & $\mathbf{8 2 3 . 4 8}$ & $\mathbf{1 , 2 0 8 . 5 7}$ & $\mathbf{2 , 3 6 7 . 4 7}$ & & \\
(B2B only) & & & & & & & \\
\hline
\end{tabular}

Table 1: Worldwide e-commerce estimates and forecasts (billion USD) (Source: UNCTAD)

\section{E-HEALTH}

Reducing healthcare expenditure as well as offering quality healthcare treatment is becoming a priority globally. Technology and automation have the potential to reduce these costs [Institute of Medicine, 2001][Wickramasinghe, 2000]; thus, e-health, which essentially involves the adoption and adaptation of e-commerce technologies throughout the healthcare industry [Eysenbach,2001], appears to be a powerful force of change for the healthcare industry worldwide.

Healthcare has been shaped by each nation's own set of cultures, traditions, payment mechanisms and patient expectations. Therefore, when looking at health systems throughout the world, it is useful to position them on a continuum ranging from high government involvement (i.e. a public healthcare system) at one extreme to little government involvement (i.e., private healthcare system) at the other extreme with many variations of a mix of private-public in between. However, given the common problem of exponentially increasing costs facing healthcare globally, irrespective of the particular health system one examines, the future of the healthcare industry will be partially shaped by commonalties such as the universal issue of escalating costs and the common forces of change including: i) empowered consumers, ii) e-health adoption and adaptability and iii) shift to focus on the practice of preventative versus cure driven medicine, as well as four key implications, namely : i) health insurance changes, ii) workforce changes and changes in the roles of stakeholders within the health system, iii) organizational changes and standardization and iv) the need for healthcare providers and administrators to make difficult, yet necessary choices regarding practice management.

E-health is a very broad term that encompasses various activities related to the use of many e-commerce technologies and infrastructures most notably the Internet for facilitating healthcare practice. The World Health Organization [WHO, 2003] a major world health body, defines e-health as "being the leveraging of the information and communication technology (ICT) to connect provider and patients and governments; to educate and inform health care professionals, managers and consumers; to stimulate innovation in care delivery and health system management; and, to improve our healthcare system". In contrast, a technologically oriented definition of e-health is offered by Intel which refers to e-health as "a concerted effort undertaken by leaders in healthcare and hi-tech industries to fully harness the benefits available through convergence of the Internet and healthcare."

Health professionals are increasingly being drawn into evaluating the Internet as a source of consumer information for health and medicine. Practitioners report that a growing number of patients arrive at their offices either with questions related to appropriate web sites to visit or a large variety of health-related content gathered from the Internet. Some of this content may prove extremely helpful to the health and/or recovery of a patient. Because the Internet has created new opportunities and challenges to the traditional healthcare information technology industry, the advent of e-health seems fitting to address both these opportunities and challenges. The new possibilities for facilitating effective healthcare delivery fall primarily into the following main categories: 1) The capability of healthcare consumers to interact with their providers online (B2C = "business to consumer"), 2) The possibility to improve healthcare institution-to-institution transmissions of data (B2B = "business to business") and 3) The new possibilities for peer-to-peer communication of healthcare consumers $(\mathrm{C} 2 \mathrm{C}=$ "consumer to consumer").

A more comprehensive definition of e-health would need to incorporate healthcare, business and technological perspectives; hence we define e-health as an emerging field at the intersection of medical informatics, technology, public health and business. Thereby, e-health entails the delivery of health services and health information through the Internet and other related e-commerce technologies. In a broader sense, the term characterizes not only a technical development, but also a state-of-mind, a paradigm shift, and a commitment for networked global thinking to improve healthcare locally, regionally, and globally by using information and communication technologies. 


\section{The Goals of E-Health}

The preceding definition of e-health is broad enough to capture the dynamic environment of the Internet and at the same time acknowledge that e-health encompasses more than just "Internet and Medicine". Thus e-health strives to achieve many goals beyond the use of the Internet. These goals, taken together perhaps best characterize what e-health is all about (or what it should be about) [JMIR, 2003]. Some of these goals of e-health include:

Efficiency - one of the promises of e-health is to increase efficiency in healthcare, thereby decreasing costs. One possible way of decreasing costs would be by avoiding duplicative or unnecessary diagnostic or therapeutic interventions, through enhanced communication possibilities between healthcare establishments, and through patient involvement [Health Technology Center, 2000]. The Internet will naturally serve as an enabler for achieving this goal in e-health.

Quality of care - increasing efficiency involves not only reducing costs, and thus is not an end in and of itself, but rather should be considered in conjunction with improving quality one of the ultimate goals of e-health. A more educated consumer (as a result of the informational aspects of e-health) would then communicate more effectively with their primary care provider which will, in turn, lead to better understanding and improved quality of care.

Evidence-based - e-health interventions should be evidence-based in the sense that their effectiveness and efficiency should not be assumed but proven by rigorous scientific evaluation and support from case histories. Web accessible case repositories facilitate the timely accessibility of such evidence and thus help in achieving the necessary support of a diagnosis or treatment decision. The evidence-based medicine goal of e-health is currently one of the most active e-health research domains, yet much work still needs to be done in this area.

Empowerment of consumers and patients - by making the knowledge bases of medicine and personal electronic records accessible to consumers over the Internet, e-health opens new avenues for patient-centered medicine, enables patient education, and thus increases the likelihood of informed and more satisfactory patient choice [Umhoff and Winn, 1999].

Education of physicians through online sources (continuing medical education) and consumers (health education, tailored preventive information for consumers) makes it easier for physicians as well as consumers to keep up to date with the latest developments in the medical areas of their respective interests. This, in turn, is likely to have a positive impact on the quality of care vis-à-vis the use of the latest medical treatments and preventive protocols.

Extending the scope of healthcare beyond its conventional boundaries, in both a geographical sense as well as in a conceptual sense leads to enabling such techniques as telemedicine and virtual operating rooms, both of which are invaluable in providing healthcare services to places where it may otherwise be difficult or impossible to do.

Ethics - e-health involves new forms of patient-physician interaction and poses new challenges and threats to ethical issues such as online professional practice, informed consent, privacy and security issues [Healthcare Advisory Board Report, 2002]. However, this is not an intrinsic feature of e-health but rather a feature of the Internet technology which is the foundation for all e-business initiatives, therefore, e-health along with e-government, e-insurance, e-banking, e-finance and eretailing must all contend with these ethical issues. Given the nature of healthcare, these issues could be more magnified.

Equity - to make healthcare more equitable is one of the aims of quality identified by the American Institute of Medicine [Institute of Medicine, 2001] generally and is one of the goals of e-health. However, at the same time there is a considerable threat that e-health, if improperly implemented and used, may deepen the gap between the "haves" and the "have-nots", hence the need for a robust framework to ensure the proper implementation of e-health initiatives. In particular, some of the key issues for equity revolve around broad access and familiarity with the technology.

Today, a large number of patients and consumers already use the Internet to retrieve health related information, to interact with health providers and even to order pharmaceutical products (for example drugstore.com). For example, it has been noted that "the number of Medline searches performed by directly accessing the database at the National Library of Medicine increased from 7 million in 1996 to 120 million in 1997, when free public access was opened; the new searches are attributed primarily to non-physicians" [JMIR, 1999]. Thus, the Internet will act as a catalyst for evidence based medicine in particular and e-health generally in two ways: First, it enables health professionals to access timely evidence. Second, it enables consumers to draw from the very same knowledgebase, leading to increased pressure on health professionals to actually use the evidence [PWC, 2003]. 


\section{Key Challenges of E-Health}

By 2005, 88.5 million adults will use the Internet to find health information, shop for health products and communicate with affiliated payers and providers through online channels, according to Cyber Dialogue [PWC, 2003]. Today, the e-health consumer demand includes the need for specific health services, such as obtaining information when faced with a newly established diagnosis.

Many key challenges must be addressed to develop optimal partnerships between consumers and other groups of healthcare stakeholders. Some of these include the need for:

- Meaningful collaboration with healthcare recipients,

- Efficient strategies and techniques to monitor patterns of Internet use among consumers,

- Preparation for upcoming technological developments,

- Balancing between connectivity and privacy,

- Better understanding of the balance between face-to-face and virtual interactions, and

- Equitable access to technology and information across the globe.

When we consider the domain of e-health at the macro level, three important issues must be carefully considered [Cyber Dialogue, 2001]; namely, Procurement, Connectivity, and Benefits. We briefly discuss each in turn

\section{E-Procurement}

Health systems must begin to contemplate how their organizations will adapt and leverage Internet-based tools to manage their medical supply chains. Procurement in healthcare supplies must move toward an e-business platform for data interchange because of the ubiquity and cost effectiveness of the Internet, the primary e-business platform. The Internet decreases many of the restrictions placed by geographic and time barriers and also facilitates the use of incorporating artificial intelligence solutions such as intelligent agents that can be deployed to hunt for best buys. Buyers and suppliers also must work together toward standardization, including the development of a universal product numbering system to facilitate e-procurement. As shown in figure 1, e-procurement of medical supplies is estimated to grow to $15 \%$ of medical supply spending in the U.S. by 2003, according to a Deutsche Bankreport [PWC, 2003].

\section{E-Procurement Expecred to Capture $15 \%$ of Market by 2003}

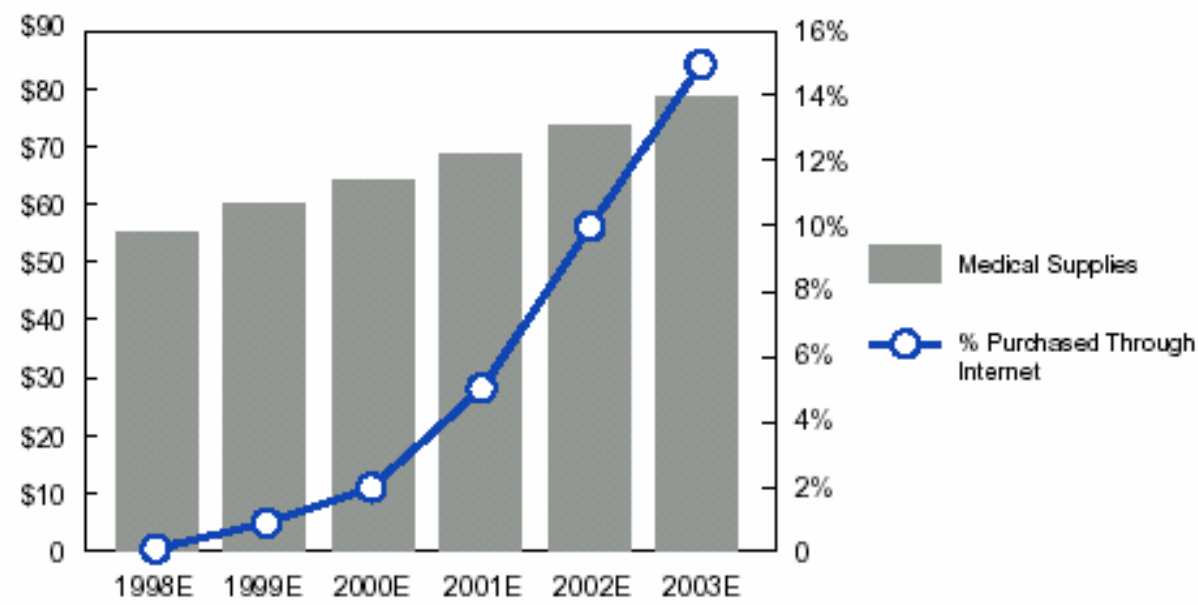

Figure 1: Estimated US medical e-procurement market sales (Source: Odyssey, 2003)

\section{E-Connectivity}

Healthcare has traditionally been locally delivered because a patient's usual first port of call is their local primary care physician. To re-orient such a tradition, connectivity companies, which on the other hand have a global rather than local focus, must be pragmatic and move in incremental steps when connecting healthcare organizations. Technology is the integral tool, but it will not achieve its full potential or live up to its promise unless healthcare organizations successfully deploy it and then track if their clinicians and administrative workers are using it effectively. To do so, managers must design processes and metrics for productivity; otherwise, it's like expecting someone to drive a car when his/her previous experience is limited to a 10 -speed bicycle. However, healthcare organizations will find that achieving web-enabled connectivity offers 
the most opportunity initially, and that other functions such as disease management, outcomes management, and demand management can be web-enabled at a later point.

Health plans and hospitals are beginning to migrate to the Internet for claims-related transactions as the first step of a broader Internet strategy. This is illustrated in figure 2. Since many organizations continue to use EDI for claims submissions, transactions surrounding claims for example eligibility, referrals, etc., these will thus logically be the first to be targeted for e-health connectivity. Those health plans that are adopting Internet connectivity for these functions view them as the foundation on which to build other Internet-enabled partnerships with patients and providers.

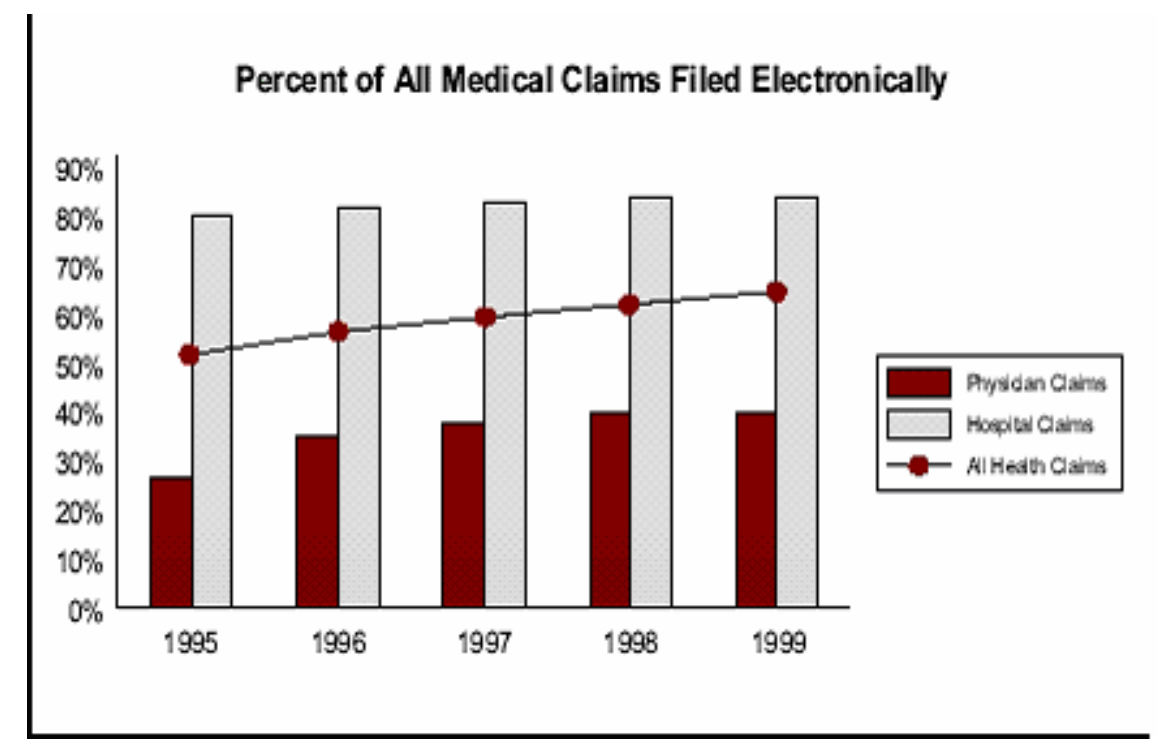

Figure 2: Increase in the percentage of electronically filed medical claims (Source: Odyssey, 2003)

The number of healthcare transactions is outpacing the growth of health spending, creating a critical need to automate the handling of such transactions; i.e. through the adoption of e-health. The number of claims submitted increased by about 7\% during the past five years, according to the Health Data Directory. In contrast, healthcare spending has increased at between 5\% and 6\% during the same period (ibid). Many of the functions associated with claims submissions and payment are repetitive tasks that are more efficiently done by computers. The most expensive processes are not the claims submissions themselves, but the tasks surrounding the claims processing, such as eligibility checks and referrals. Coupled with increasing labor shortages, there is an urgent need for healthcare organizations to re-engineer their processes.

Health plans must understand physicians' needs when designing Internet-based solutions and that first-mover advantage isn't as important as a system that works. Many non-healthcare organizations have benefited from first-mover advantage in adopting e-commerce initiatives, which means they had access to the most capital, the best partners and could establish solid brand recognition. However, this is not necessarily an appropriate strategy for healthcare as healthcare is more pragmatic and a "show-me" industry where successful models replicate specialty by specialty.

\section{E-Benefits}

American industry has discovered the benefits of e-business. Like the conundrum of the chicken-and-the-egg, many employers and health plans are awaiting the development and implementation of e-benefits and e-insurance products. Health plans don't want to deliver web-based products if employers aren't ready to use them. Employers can't deliver e-benefits products until health plans develop them. However, starting with online benefits enrollment, this aspect of e-health is evolving in stages. A byproduct of this evolution is the fusion of employee responsibility and empowerment; hence employers will gradually cede more control for health benefits to employees themselves. One of the primary drivers of ebenefits is delivery of self-service capabilities in which employees can customize their own insurance plans and have ready access to them, just as they do with their brokerage accounts. By putting this information at their fingertips, employees may become more fiscally responsible about those benefits. Ultimately, they may want complete control over more and more aspects of their benefits. To empower employees toward that end, some employers will have to embrace a defined contribution model, also called self-directed or consumer-directed health plans. This builds on the findings of the report "Defined Contribution Healthcare", which specifically discusses the various models of defined contribution health plans 
(ibid). The national research conducted by PricewaterhouseCoopers (ibid) indicates that few employers are willing to adopt self-directed, otherwise known as defined contribution, health plans today, but will more likely move incrementally toward web-enabling benefits processing and hence shifting more responsibility to employees. As employers adopt more e-health initiatives and web-enabled functions, they will move more responsibility for choices to employees. As that balance of responsibility tips towards the employees, employers will need to assess how ready their workforce is to accept increasing levels of responsibility.

If a significant portion of the healthcare insurance market moves to self-directed and Internet health benefit accounts, physicians may be most directly and adversely affected. Some contribution health plans have medical savings accounts as a centerpiece. In these accounts, employees pay out of a medical savings account for routine expenses up to $\$ 1,500$ or $\$ 2,000$. Physicians will need to contend with patients who are paying cash for their visits and who may shop around for the best value. Figure 3 shows the statistics of the probability of customers using the Internet when they have accessibility to PCs.

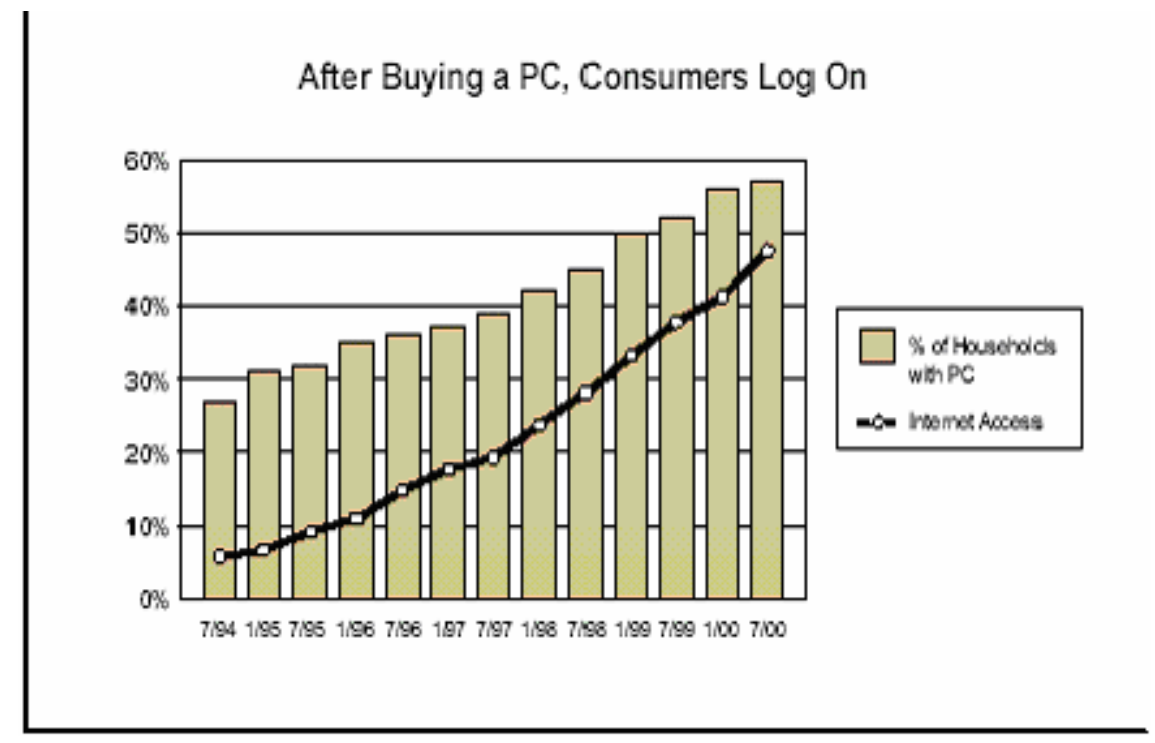

Figure 3: Internet usage among customers (Source: Odyssey, 2003)

As the utility function of benefits moves toward the Internet, insurers must then develop products that serve new and existing customers such as e-quote products that assist brokers and other intermediaries. Further, they also must follow the changing needs of consumers whose response to defined contribution health products is to date largely untested. In summary then, the effective addressing of the key e-health issues of procurement, connectivity and benefits requires standardization, a pivotal pre-requisite for the implementation of successful e-health initiatives. Without such standardization the exchange of documents and other procurement information, connectivity and e-commerce enabled benefits clearly become more problematic. Unfortunately, standardization is woefully lacking in too many areas of healthcare let alone e-health. Given the opportunities for e-health to benefit various aspects of healthcare and the far reaching impact of any such e-health initiative, it becomes imperative then to have frameworks and models that not only bring to the forefront the key e-health issues but also provide guidelines for how to effectively bring to bear successful e-health initiatives for all healthcare organizations.

\section{A FRAMEWORK FOR ASSESSING E-HEALTH POTENTIAL}

We propose the framework shown in figure 4 to assess the e-health potential and preparedness of countries. Healthcare polices are generally developed to a large extent at a macro, country level and thus we believe it is also necessary when looking at e-health to first take a macro perspective and analyze the level of the country in terms of embracing e-health. The framework highlights the key elements that are required for successful e-health initiatives and therefore provides a tool that allows analysis beyond the quantifiable data into a systematic synthesis of the major impacts and pre-requisites. The framework contains four main pre-requisites, four main impacts, and the implications of these pre-requisites and impacts to the goals of e-health. By examining both the pre-requisites and the impacts we can assess the potential of a country and its preparedness for e-health as well as its ability to maximize the goals of e-health. We discuss the prerequisites next. 


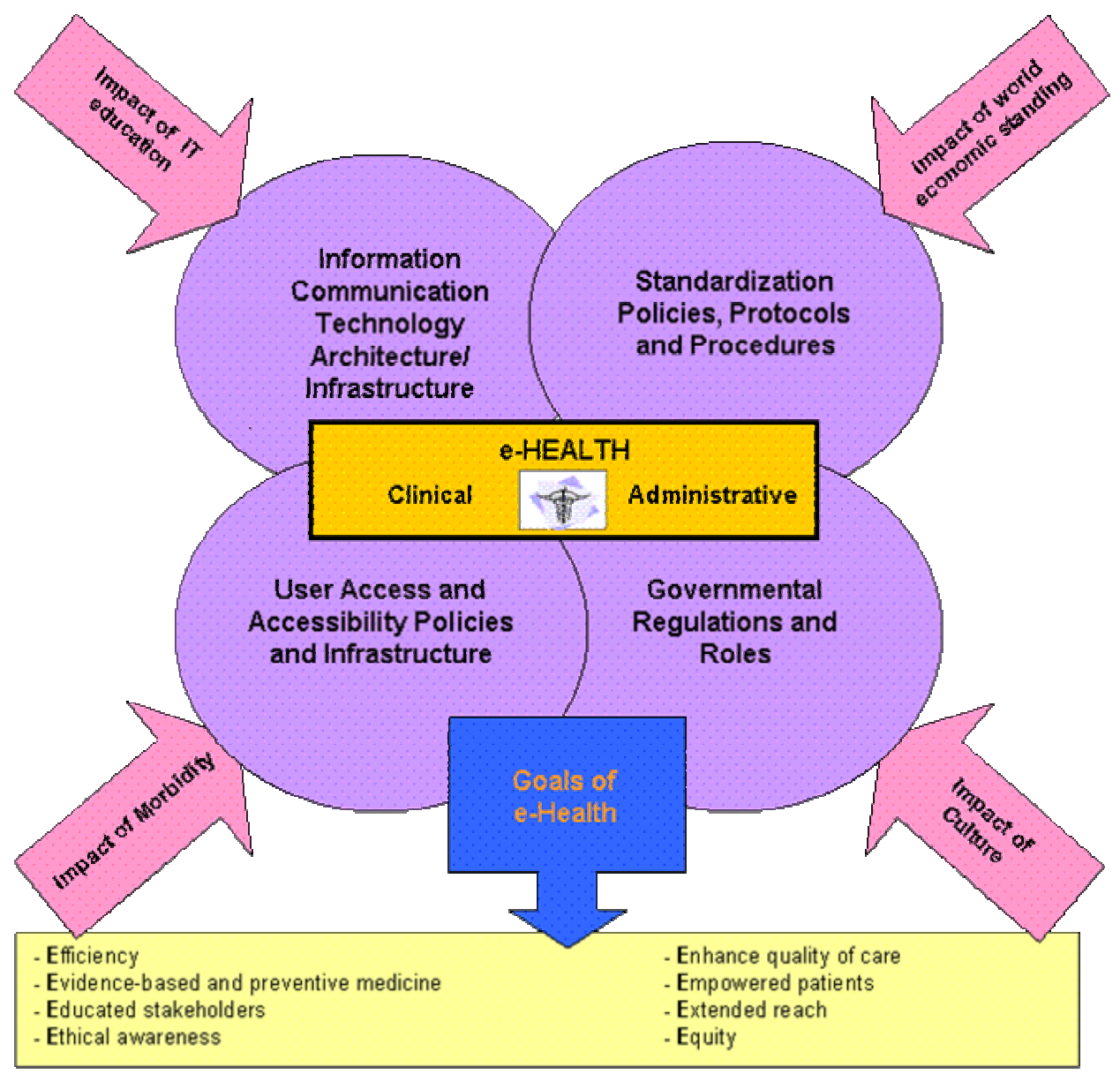

Figure 4: Framework for assessing a country's/region's e-health potential

\section{Prerequisites for e-Health}

As can be seen in figure 4 above the four critical pre-requisites for any successful e-health initiative include information communication technology architecture/infrastructure, standardized policies, protocols and procedures, user access and accessibility policies and infrastructure, and finally government regulation and control. These will now be briefly discussed in turn.

\section{Information communication technology (ICT) architecture/ infrastructure}

The generic architecture for most e-health initiatives is depicted in figure 5. To support such a client-server architecture special attention must be paid to the ICT infrastructure. The ICT infrastructure includes phone lines, fiber trunks and submarine cables, T1, T3 and OC-xx, ISDN, DSL and other high-speed services used by businesses as well as satellites, earth stations and teleports. A sound technical infrastructure is an essential ingredient to the undertaking of e-health initiatives by any nation. Such infrastructures should also include telecommunications, electricity, access to computers, number of Internet hosts, number of ISP's (Internet Service Providers) and available bandwidth and broadband access. To offer a good multimedia content and thus provide a rich e-health experience, one would require a high bandwidth. ICT considerations are undoubtedly one of the most fundamental infrastructure requirements.

Networks are now a critical component of the business strategies for organizations to compete globally. Having a fast microprocessor-based computer at home has no meaning unless you have high bandwidth based communication infrastructure available to connect computers with the ISP. With the explosion of the Internet and the advent of e-commerce, global networks need to be accessible, reliable, and fast to participate effectively in the global business environment. Telecommunications is a vital infrastructure for Internet access and hence for e-commerce. One of the pioneering countries in establishing a complete and robust e-health infrastructure is Singapore which is in the process of wiring every home, office and factory up to a broadband cable network which will cover $98 \%$ of Singaporean homes and offices (ibid). 


\section{Client-Server Computing Paradigm based Architecture}

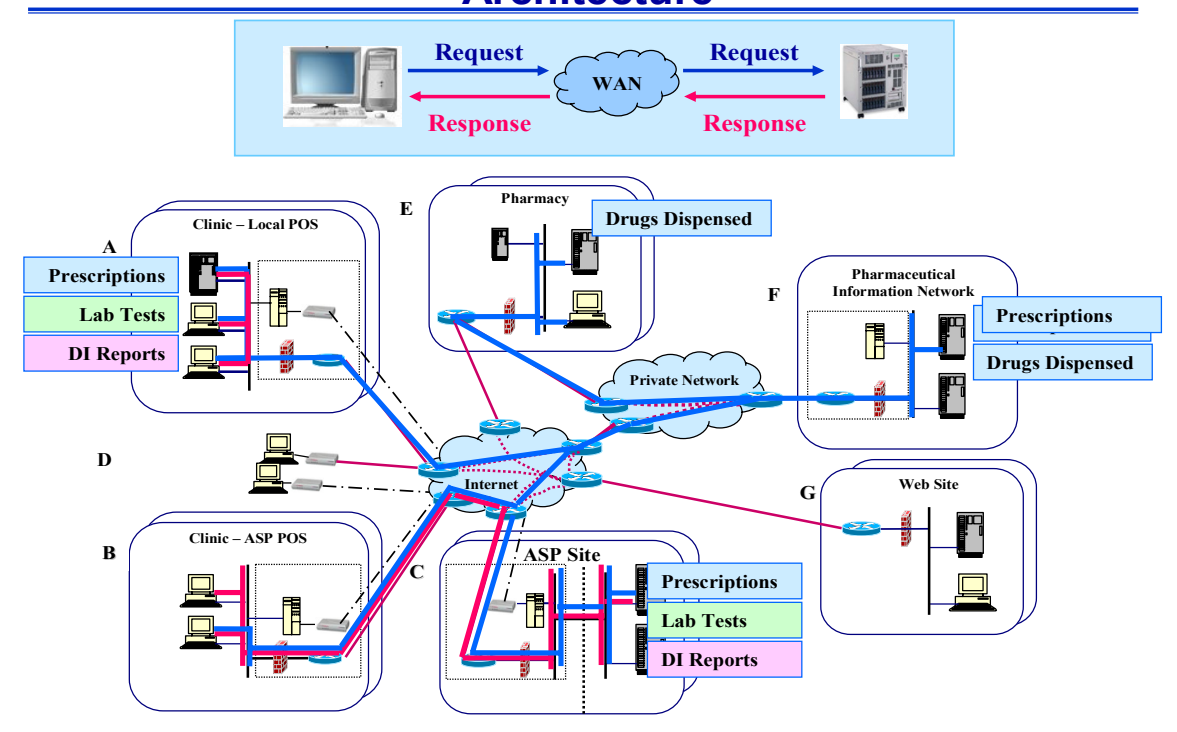

Figure 5: The essential ICT architecture for e-health

\section{Standardization policies, protocols and procedures}

e-Health by definition spans many parties and geographic dimensions. To enable such a far reaching coverage, significant amounts of document exchange and information flows must be accommodated. Standardization is the key for this. Once a country decides to undertake e-health initiatives, standardization polices, protocols and procedures must be developed at the outset to ensure the full realization of the goals of e-health. Fortunately, the main infrastructure of e-health is the Internet which imposes the most widely and universally accepted standard protocols such as TCP/IP and http. It is the existence of these standard protocols that has led to the widespread adoption of the Internet for e-commerce applications.

The transformation to e-health by any country cannot be successfully attained without the deliberate establishment of standardization policies, protocols and procedures which play a significant role in the adoption of e-health and the reduction of many structural impediments [Samiee, 1998].

\section{User access and accessibility policies and infrastructure}

Access to e-commerce is defined by the WTO (World Trade Organization) as consisting of two critical components 1) access to Internet services and 2) access to e-services [Panagariya, 2000]; the former deals with the user infrastructure, while the latter pertains to specific commitments to electronically accessible services. The user infrastructure includes number of Internet hosts and number of web sites, web users as a percent of the population as well as ISP availability and costs for consumers, PC penetration level etc. Integral to user infrastructure is the diffusion rate of PCs and Internet usage. The United States and the United Kingdom have experienced the greatest penetration of home computers [Samiee, 1998]. For developing countries such as India and China there is however, a very low PC penetration and tele-density. In such a setting it is a considerable challenge then to offer e-health, since a large part of the population is not able to afford to join the ecommerce bandwagon. Countries, thus have to balance local call charges, rentals, subscription charges etc., otherwise the majority of citizens will find these costs a disincentive. This is particularly significant for developing and emerging nations where access prices tend to be out of reach for most of the population. Upcoming new technologies hold the promise to increase the connectivity as well as affordability level and developing countries will need to seriously consider these technologies. In addition to access to PCs and the Internet, computer literacy is important and users must be familiar not only with the use of computers and pertinent software products but also the benefits and potential uses of the Internet and World Wide Web (ibid).

\section{Governmental regulation and control}

The key challenges regarding e-health use include; 1) cost effectiveness; i.e. less costly than traditional helathcre delivery, 2) functionality and ease of use, i.e., they should enable and facilitate many uses for physicians and other healthcare users by combining various types and forms of data as well as be easy to use; and 3) they must be secure. One of the most significant legislative regulations in the US is the Health Insurance Portability and Accountability Act (HIPAA) [Protegrity, 2001]. Table 2 summarizes the key HIPAA requirements. 


\section{Extracts from HIPAA Security Requirements}

- Establishment of trust partnership agreements with all business partners

- Formal mechanisms for accessing electronic health records

- Procedures and policies to control access of information

- Maintaining records of authorizing access to the system

- Assuring that system users receive security awareness training and the training procedures are periodically reviewed and updated

- Maintaining security configuration including complete documentation of security plans and procedures, security incident reporting procedure

- Communication and network control including maintaining message integrity, authenticity and privacy. Encryption of messages advocated for open network transmission portion of the message

- Data authentication to ensure that data is not altered or destroyed in an unauthorized manner

Table 2: HIPAA requirements (Source: Protegrity, 2003)

Given the nature of healthcare and the sensitivity of healthcare data and information, it is incumbent on governments not only to mandate regulations that will facilitate the exchange of healthcare documents between the various healthcare stakeholders but also to provide protection of privacy and the rights of patients. Some countries, such as China and Singapore, even control access to certain sites for moral, social and political reasons while elsewhere trans-national data flows are hindered by a plethora of regulations aimed at protecting domestic technology and related human resource markets [Samiee, 1998][Goff,1992][Gupta,1992]. Irrespective of the type of healthcare system; i.e., whether 100\% government driven, $100 \%$ private or a combination thereof, it is clear that some governmental role is required to facilitate successful ehealth initiatives. The significance of the preceding four pre-requisites on e-health initiatives will be modified by the impacts of IT education, morbidity, cultural dimensions and world economic standing as elaborated upon below.

\section{Key Impact of E-Health}

Figure 4 also highlights four key impacts of e-health (depicted in rectangular boxes) which we now discuss in turn.

\section{Impact of IT education}

A sophisticated, well educated population boosts competition and hastens innovation. According to Michael Porter, one of the key factors to a country's strength in an industry is strong customer support [Porter,1990]. Thus, a strong domestic market leads to the growth of competition which leads to innovation and the adoption of technology enabled solutions to provide more effective and efficient services such as e-health and telemedicine. As identified earlier, the health consumer is the key driving force in pushing e-health initiatives. We conjecture that a more IT educated healthcare consumer would then provide stronger impetus for e-health adoption.

\section{Impact of morbidity rate}

There is a direct relationship between health education and awareness and the overall health standing of a country. Therefore, a more health conscious society, which tends to coincide with a society that has a lower morbidity rate, is more likely to embrace e-health initiatives. Furthermore, higher morbidity rates tend to indicate the existence of more basic health needs [WHO, 2003] and hence treatment is more urgent than the practice of preventative medicine and thus e-health could be considered an unrealistic luxury and in some instances such as when a significant percentage of a population is suffering from malnutrition related diseases is even likely to be irrelevant at least in the short term. Thus, we conjecture that the modifying impact of morbidity rate is to prioritize the level of spending on e-health versus other basic healthcare needs.

\section{Impact of cultural/social dimensions}

Healthcare has been shaped by each nation's own set of cultures, traditions, payment mechanisms and patient expectations. While the adoption of e-health, to a great extent, dilutes this cultural impact, social and cultural dimensions will still be a moderating influence on any countries e-health initiatives. Another aspect of the cultural/social dimension relates to the presentation language of the content of the e-health repositories. The entire world does not speak English so the e-health solutions have to be offered in many other languages. The e-health supporting content in web servers/sites must be offered in 
local languages, supported by pictures and universal icons. This becomes a particularly important consideration when we look at the adoption and diffusion of evidence-based medicine as it will mean that much of the available evidence and case study data will not be easily accessible globally due to language barriers.

Therefore, for successful e-health initiatives it is important to consider cultural dimensions. For instance, an international e-commerce study by International Data Corp. indicates that web surfing and buying habits differ substantially from country to country [Wilson, 1999] and this would then have a direct impact on their comfort to use e-commerce generally and e-health in particular, specially as e-health addresses a more fundamental need. Hence, the adoption of ehealth is directly related to ones comfort with using the technology and this in turn is influenced in a major way by cultural dimensions. Also connected with cultural aspects is the relative entrepreneurial spirit of a country. For example, a study [Hofstede, 1980], indicates that in a cultural context, Indians score high on "uncertainty avoidance" criteria when compared to their Western counterparts. As a result, for example Indians do not accept change very easily and are hostile towards innovation. This then would potentially pose a challenge to the starting up of e-health initiatives whose success depends on wide spread adoption for their technological innovations. Thus, we conjecture that fear of risk and absence of an entrepreneurial mindset as well as other cultural/social dimensions can also impact the success of e-health initiatives in a given country.

\section{Impact of world economic standing}

Economies of the future will be built around the Internet. All governments are very aware of the importance and critical role that the Internet will play on a country's economy. This makes it critical that appropriate funding levels and budgetary allocations become a key component of governmental fiscal policies so that such initiatives will form the bridge between a traditional healthcare present and a promising e-health future. Thus, the result of which would determine success of effective e-health implementations and consequently have the potential to enhance a country's economy and future growth.

The World Economic Forum's Global competitiveness ranking measures the relative global competitiveness of a country. This ranking takes into account factors such as physical infrastructure, bureaucracy and corruption. Thus we conjecture that when weak physical infrastructure is combined with high levels of bureaucracy and corruption this will lead to significant impediments to the establishment of successful e-health initiatives. In developing its e-health initiative, a good first step for any country is to assess its standing with respect to the four pre-requisites and four impacts discussed above. In this way it will be possible to evaluate its preparedness with respect to these parameters and consequently devise appropriate policies and strategies for an effective and successful e-health initiative. In the following section, we will attempt to provide a guideline that will facilitate such an evaluation.

\section{E-HEALTH PREPAREDNESS GRID}

By taking the four main pre-requisites as well as the four major impacts identified in our framework in figure 4; namely, the information communication technology infrastructure, the standardization policies, protocols and procedures, the user access and accessibility policies and infrastructures, governmental regulations and role as well as the impact of IT education, the impact of morbidity rate, the impact of world economic standing and the impact of cultural/social dimensions, we develop a grid for assessing e-health preparedness (figure 6) in which we can plot various countries with respect to these key parameters. The grid consists of four quadrants that represent the possible states of preparedness with respect to the key parameters for e-health success. The low preparedness quadrant identifies situations that are low with respect to all four prerequisites for e-health potential. The medium preparedness quadrant identifies two symmetric situations; namely, a combination of high and low positioning with respect to the four pre-requisites for e-health potential. Finally, the high preparedness quadrant identifies situations that are high with respect to all four pre-requisites for e-health potential. This grid not only shows the possible positioning of a given country with respect to its e-health preparedness (i.e. low, medium or high) but also the path it must take, and more specifically the pre-requisite factors it must focus on, to migrate to the ideal state of preparedness; namely, being high with respect to all four pre-requisites. The grid also underscores the moderating role of the four impacts irrespective of the relative positioning on the state of preparedness of a given country. 


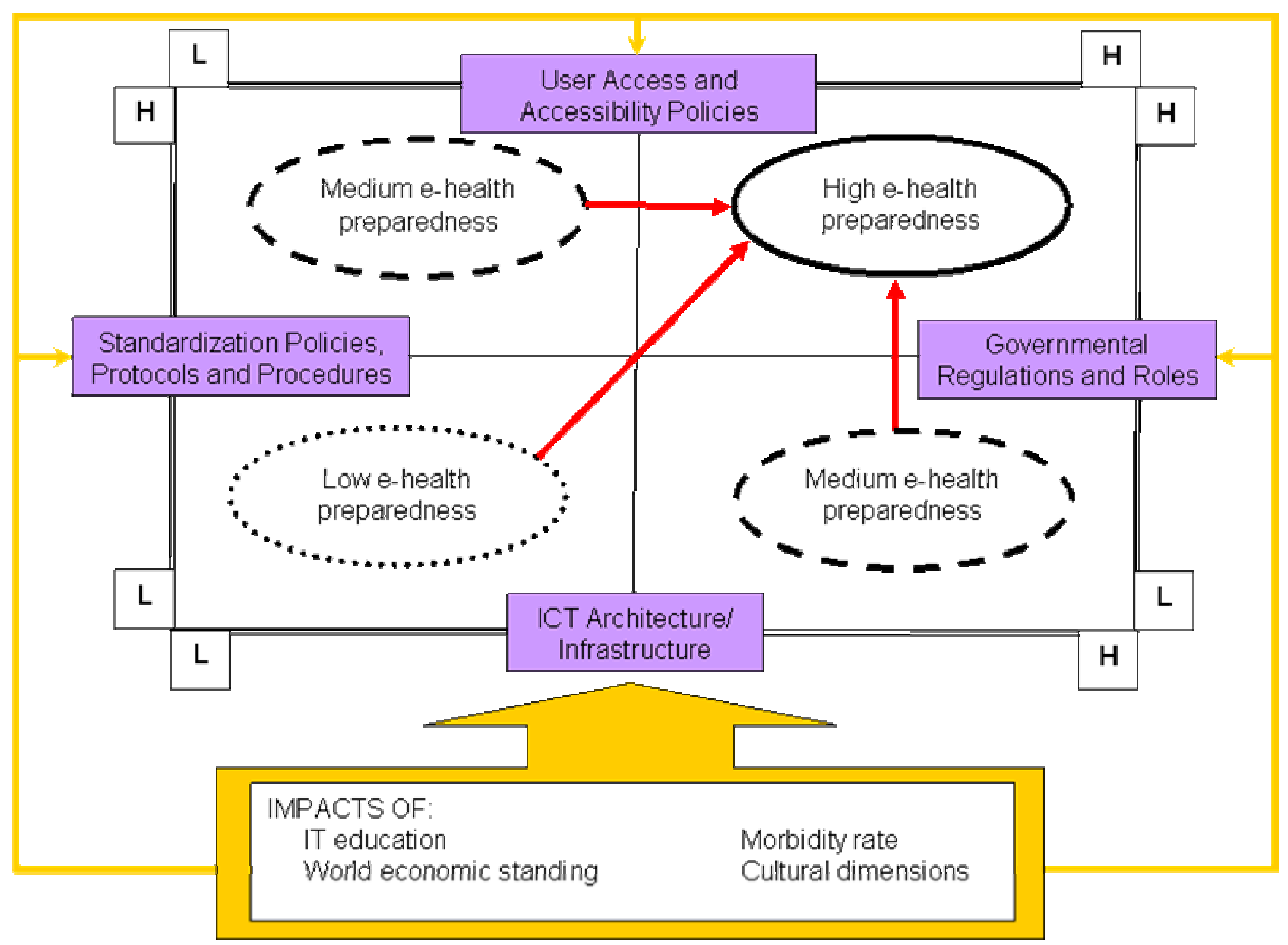

Figure 6. e-Health preparedness grid

\section{DISCUSSION}

From the e-Health Preparedness Grid in figure 6 we can see several implications. For countries which are low with respect to all four of the e-health pre-requisites, much preparatory work is required for these countries to be e-health ready and thereby fully realize the goals of e-health (i.e. efficiency, evidence-based and preventive medicine, educated stakeholders, ethical awareness, enhanced quality care, empowered patients, extended reach and equity). For countries which map to the medium preparedness quadrant, more emphasis is needed on upgrading the deficiencies which cause them to score low on the respective pre-requisites while they continue to maintain their high status on the pre-requisites on which they currently rank high so that the full benefits of their e-health initiatives can be realized. Countries noted for being pioneers and leaders in the area of e-commerce generally as well as e-health in particular would be expected to map on the high quadrant. The challenge for these countries would be to maintain their high status with respect to all the pre-requisites. Furthermore, organizations within these countries can also begin to structure their respective e-health initiatives; i.e. micro level issues based on the key macro level issues which both the integrative framework and e-health preparedness grid identify.

It is important to note that irrespective of their positioning on the preparedness grid (i.e. low, medium or high), all countries must take into account the moderating effect of the four impacts on their e-health initiatives. If countries ignore the moderating effect of these four impacts it will not be possible to fully realize a successful e-health initiative (and thus would not be able to fully realize the benefits of e-health), even if a country maps into the high preparedness quadrant. Finally, we conjecture that the type of health system vis-à-vis the public/private continuum, would not significantly impact the positioning of a country into a given quadrant. The successful experience of Singapore, albeit an isolated case, somewhat lends credence to our grid as it would mostly fit the profile of a country in the high preparedness quadrant and thus would be expected to successfully undertake an e-health initiative as the PWC report indicates [PWC, 2003] Clearly additional empirical validation of our e-health preparedness framework is required which we leave to future studies. 


\section{CONCLUSION}

E-commerce, as noted by the UN Secretary General's address, is an important aspect of business in today's $21^{\text {st }}$ century. No longer then is it a luxury for nations, rather it is a strategic necessity in order for countries to achieve economic and business prosperity as well as social viability. One of the major areas within e-commerce that has yet to reach its full potential is e-health. This is due to the fact that healthcare generally has been slow in adopting information technologies. Furthermore, there is a shortage of robust frameworks that may be used as guidelines for assessing countries e-health preparedness and identifying the key areas and deficiencies that need to be addressed in order for successful e-heath initiatives to ensue. In addition, e-health is more than a technological initiative; rather it also requires a major paradigm shift in healthcare delivery, practice and thinking. We have attempted to address this gap by developing a framework that identifies the major factors involved in assessing the e-health preparedness of countries and thereby, facilitating countries in focusing their efforts on the relevant issues that must be addressed in order that successful e-health initiatives follow (the goals of e-health are realized). An outcome from our analysis indicates that the relative healthcare system (i.e. whether government driven, public or two tier) would appear to have less significance in establishing successful e-health initiatives. The first step in the development of any viable e-health strategy is to make an assessment of the current state of e-health preparedness and then how to either move to a state of higher preparedness (i.e., the high quadrant) or focus on maintaining a current high quadrant status - both of these are identified through the use of our framework and thus its value. Finally, we note that with respect to our framework other parameters also exist and could also be considered important, perhaps even as important as the ones we used. However, we believe that the framework will still function the same way (i.e., provide a useful tool for any country trying to determine and develop a successful e-health initiative), irrespective of the number of parameters; in this regard we preferred simplicity over complexity.

\section{REFERENCES}

1. UNCTAD. United Nations Conference on trade and Development $2002 \mathrm{http}: / / \mathrm{r} 0$.unctad.org/ecommerce/ecommerce en

2. Institute of Medicine. Crossing the Quality Chasm -a New Health System for the 21 st Century Committee on Quality of Health Care in America Institute of Medicine, National Academy Press, Washington, D.C.

3. Wickramasinghe, N 2000 "IS/IT as a Tool to Achieve Goal Alignment: a theoretical framework" International J. Healthcare Technology Management Vol2. No. 1/2/3/4/. pp. 163-180.

4. World Health Organization 2003. http://www.emro.who.int/ehealth/

5. JMIR. Journal of Medical Internet Research @ http://www.jmir.org

6. Sieving PC Factors Driving the Increase in Medical Information on the Web - One American Perspective. J Med Internet Res 1999;1@www.jmir.org/1999/1/e3

7. PricewaterhouseCoopers Healthcare Practice@www.pwchealth.com

8. Cyber Dialogue. Online Health Information Seekers Growing Twice as Fast as Online Population. Cyber Dialogue, May 23, 2000.

9. Cyber Dialogue. The Higher the Connection Speed, the Higher the Value: Broadband Use as an Indication of Value amongst e-Health Users. Cyber

10. Odyssey Research. Dialogue Cybercitizen Health Trend Report, Yr2001, No.49, Odyssey Research @ www.odysseyresearch.org

11. Samiee, S 1998 “The Internet and International Marketing: Is There a Fit?” Journal of Interactive Marketing, vol 12 no. 4 pp.5-20

12. Panagariya, A. E-commerce, WTO and developing countries. The World Economy, 23, 8, (2000), 959-978.

13. Protegrity. Health Insurance Portability and Accountability Act (HIPPA) Privacy Compliance Executive Summary, Protegrity Inc. May 2001.

14. Goff, L. 1992 "Patchwork of Laws Slows EC data Flow" Computerworld, 26, 15, 80.

15. Gupta, U. 1992 "Global Networks: Promises and Challenges" Information Systems Management 9, 4 28-32.

16. Porter, M. 1990 The Competitive advantage of Nations, Free Press New York.

17. Wilson, T. Not A Global Village After All? -- Consumer Behavior Varies Widely By Country. Internetweek, 792, (1999) 13.

18. Hofstede, G. 1980 Culture's Consequences, International Work Related Values, Sage Publishing Beverly Hills.

19. Al-Qirim, N. (2004) "Telemedicine: Building Knowledge-Based Tele-health Capability in New Zealand” in Eds Wickramasinghe, N., J. Gupta and S. Sharma Creating Knowledge Based Healthcare Organizations Idea Group Publishing forthcoming

20. Alvarez, R.C. (2002) The promise of e-Health - a Canadian perspective, eHealth International, 1 (1): 4

21. Eysenbach, G (2001) Journal of Medical Internet Research 3(2): e20

22. Healthcare Advisory Board Report (2002) "Use of Hospital Web Sites to Engender Community Loyalty, January 2000.

23. Health Technology Center (2000) A survey conducted for the Health Technology Center (HealthTech) by Harris Interactive in cooperation with Pricewaterhouse Coopers and the Institute for the Future (IFTF). http://www.ncddr.org/cgi-bin/goodbye.cgi?url=http://www.healthtechcenter.org

24. Umhoff, B., and Winn, J. (1999) “The Healthcare Profit Pool: Who Stands to Gain and Lose in the Digital Economy," Health Forum Journal, May 1, 1999. 